\title{
Jet Theoretical Yang-Mills Energy in the Geometric Dynamics of 2D-Monolayer
}

\author{
M. Neagu ${ }^{1}$, N.G. Krylova ${ }^{2}$, H.V. Grushevskaya ${ }^{3}$
}

\begin{abstract}
Langmuir-Blodgett films (LB-films) consist from few LB-monolayers which are high structured nanomaterials that are very promising materials for applications. We use a geometrical approach to describe structurization into LB-monolayers. Consequently, we develop on the 1 -jet space $J^{1}\left([0, \infty), \mathbb{R}^{2}\right)$ the single-time Lagrange geometry (in the sense of distinguished (d-) connection, d-torsions and an abstract anisotropic electromagnetic-like d-field) for the Lagrangian governing the 2D-motion of a particle of monolayer. One assumed that an expansion near singular points for the constructed geometrical Lagrangian theory describe phase transitions to LB-monolayer. Trajectories of particles in a field of the electrocapillarity forces of monolayer have been calculated in a resonant approximation utilizing some Jacobi equations. A jet geometrical Yang-Mills energy is introduced and some computer graphic simulations are exposed.
\end{abstract}

${ }^{1}$ Department of Mathematics and Informatics, University Transilvania of Braşov, 50 Iuliu Maniu Blvd., 500091 Braşov, ROMANIA, E-mail: mircea.neagu@unitbv.ro

${ }^{2}$ Physics Faculty, Belarusian State University, 4 Nezavisimosti Ave., 220030 Minsk, BELARUS, E-mail: nina-kr@tut.by

${ }^{3}$ Physics Faculty, Belarusian State University, 4 Nezavisimosti Ave., 220030 Minsk, BELARUS, E-mail: grushevskaja@bsu.by

Mathematics Subject Classification (2010): 53C60, 53C80, 81T13.

Key words and phrases: 2D-monolayer Lagrangian, cannonical nonlinear connection, Cartan linear connection, d-torsions, abstract geometrical Yang-Mills energy, Jacobi equations.

\section{Introduction}

LB-films are formed at sequential transfer of LB-monolayers of amphiphilic molecules on a solid support. The LB-monolayer having high-ordered structure self-organizes on a surface of liquid subphase at its compressing by a barrier. LB-technique allows to form films containing nanosize objects (quantum dots, nanotubes, nanowires, nanorods), which become ordered in the process of monolayer formation. The resulting LB-films possess unique properties which can not be obtained in "bulk", as they reveal nanoscale effects. Such objects find wide applications in optics and nanoelectronics [1, 9]

In the process of LB-monolayer formation on a subphase surface one observes a phase transition of the first order from 2-dimensional gas up to condense state of monolayer. Up today, a theoretical description of the phase transition has been performed with the use of the Landau theory [7. Nevertheless, such phenomenological description does not allow to obtain some experimentally observable characteristics of structurization process. It was also shown that the structurization is observed in quasi-equilibrium state of monolayer at definite values of compressing speed whereas at intermediate values the phase transition is not observed [6. At this, typical dependence of surface tension $P$ upon the surface area $S$ was observed for such values of tightening speed when the compression process occurs far beyond the equilibrium. For the correct description of such peculiarities of the process it is necessary to use kinetic and dynamical models

In the paper [5] an examination of LB-monolayer structurization process using differential geometry methods has been proposed and the corresponding 3D Finsler metric of space in which a particle of monolayer is moving has been constructed. An obtained Lagrangian will be used further.

Let us start with the usual physical time defined by the Euclidian manifold $\left(T=[0, \infty), h_{11}(t)=1\right)$, whose Christoffel symbol is $\kappa_{11}^{1}(t)=0$. Let us also consider the plane manifold $\mathbb{R}^{2}$, having the polar 
coordinates $(r, \varphi)$, where $r>0$ and $\varphi \in[0,2 \pi)$. Let us construct the 1 -jet vector bundle $J^{1}\left(T, \mathbb{R}^{2}\right) \rightarrow \mathbb{R} \times \mathbb{R}^{2}$, locally endowed with the coordinates $\left(t, x^{1}, x^{2}, y_{1}^{1}, y_{1}^{2}\right):=(t, r, \varphi, \dot{r}, \dot{\varphi})$. We remind that on the 1 -jet space $J^{1}\left(T, \mathbb{R}^{2}\right)$ a local transformation of coordinates is given by the rules (the Einstein convention of summation is used throughout this work): 1

$$
\widetilde{t}=\widetilde{t}(t), \quad \widetilde{x}^{q}=\widetilde{x}^{q}\left(x^{s}\right), \quad \widetilde{y}_{1}^{q}=\frac{\partial \widetilde{x}^{q}}{\partial x^{s}} \frac{d t}{d \widetilde{t}} \cdot y_{1}^{s},
$$

where $\tilde{d t} / d t \neq 0$ and $\operatorname{rank}\left(\partial \widetilde{x}^{p} / \partial x^{q}\right)=2$. According to Olver's opinion [11, we believe that the 1-jet spaces are natural houses for the study of many physical theories. For such a reason, using the special function

$$
f(z) \stackrel{\text { def }}{=}-\int_{-z}^{\infty} \frac{e^{-t}}{t} d t
$$

we study the 2D-motion of a particle of monolayer governed by the jet Lagrangian function $L: J^{1}\left(T, \mathbb{R}^{2}\right) \rightarrow \mathbb{R}$ defined by

$$
L(t, r, \dot{r}, \dot{\varphi})=\frac{m}{2} \dot{r}^{2}+\frac{m r^{2}}{2} \dot{\varphi}^{2} \underbrace{-p r^{5}|V| e^{\frac{2|V| t}{r}} \cdot \dot{r}^{-1}+U(t, r)}_{U_{s}^{\prime \prime}(t, r)},
$$

where we have the following physical meanings: $\bullet m$ is the mass of the particule; $\bullet V$ is the LB-monolayer compressing rate; $\bullet p$ is a constant monolayer parameter given by the physical formula

$$
p=\frac{\pi^{2} q^{2}}{\varepsilon \varepsilon_{0}} \frac{\rho_{0}^{2}}{R_{0}^{2}}
$$

- $U_{s}(t, r)$ is an electrocapillarity potential energy including the monomolecular layer function

$$
\begin{aligned}
U(t, r)= & p\left\{\left[-\frac{4}{3} r^{5}+\frac{16}{15}(|V| t) r^{4}+\frac{1}{30}(|V| t)^{2} r^{3}+\frac{1}{45}(|V| t)^{3} r^{2}+\right.\right. \\
& \left.\left.+\frac{1}{45}(|V| t)^{4} r+\frac{2}{45}(|V| t)^{5}\right] e^{\frac{2|V| t}{r}}-\frac{4}{45} \frac{(|V| t)^{6}}{r} f\left(\frac{2|V| t}{r}\right)\right\} .
\end{aligned}
$$

The differential geometry (in the sense of nonlinear connections, Cartan linear connections, d-torsions, d-curvatures etc.) produced by an arbitrary jet Lagrangian function $L: J^{1}\left(\mathbb{R}, M^{n}\right) \rightarrow \mathbb{R}$ is now completely done by Balan and Neagu in the monograph [3]. The geometrical ideas from [3] are similar (but however distinct ones) to those exposed by Miron and Anastasiei in the classical Lagrangian geometry on tangent bundles (see [8]). More accurately, these jet geometrical Lagrangian ideas were initiated by Asanov in [2] and developed further in multi-parameter way in the book [10, and in single-time way in [3]. In such a

context, this paper is devoted to the development on the 1-jet vector bundle $J^{1}\left(T, \mathbb{R}^{2}\right)$ of the single-time Lagrange geometry (see [3]) produced by the 2D-monolayer Lagrangian (1.2).

\section{The canonical nonlinear connection}

The local fundamental metrical d-tensor produced by the 2D-monolayer Lagrangian (1.2) is given by formula

$$
g_{i j}=\frac{1}{2} \frac{\partial^{2} L}{\partial y_{1}^{i} \partial y_{1}^{j}} .
$$

By direct computations, the fundamental metrical d-tensor $g_{i j}$ has the matrix form

$$
g=\left(\begin{array}{ll}
g_{11} & g_{12} \\
g_{21} & g_{22}
\end{array}\right)=\left(\begin{array}{cc}
\frac{m-2 p r^{5}|V| e^{\frac{2|V| t}{r}} \cdot \dot{r}^{-3}}{2} & 0 \\
0 & \frac{m r^{2}}{2}
\end{array}\right)
$$

\footnotetext{
${ }^{1}$ Throughout this paper the Latin letters $i, j, k, l, q, s, \ldots$ take values in the set $\{1,2\}$.
} 
In order to have $\operatorname{det} g \neq 0$, we suppose that $g_{11} \neq 0$. Consequently, the matrix $g=\left(g_{i j}\right)$ admits the inverse $g^{-1}=\left(g^{j k}\right)$, whose entries are

$$
g^{-1}=\left(\begin{array}{cc}
g^{11} & g^{12} \\
g^{21} & g^{22}
\end{array}\right)=\left(\begin{array}{cc}
\frac{2}{m-2 p r^{5}|V| e^{\frac{2|V| t}{r}} \cdot \dot{r}^{-3}} & 0 \\
0 & \frac{2}{m r^{2}}
\end{array}\right) .
$$

Using a general formula from monograph [3], we find the following geometrical result:

Proposition 2.1 For the 2D-monolayer Lagrangian (1.2), the energy action functional

$$
\mathbf{E}(t, r(t), \varphi(t))=\int_{a}^{b} L d t=\int_{a}^{b}\left[\frac{m}{2}\left(\frac{d r}{d t}\right)^{2}+\frac{m r^{2}}{2}\left(\frac{d \varphi}{d t}\right)^{2}-p r^{5}|V| e^{\frac{2|V| t}{r}} \cdot\left(\frac{d r}{d t}\right)^{-1}+U(t, r)\right] d t
$$

produces on the 1 -jet space $J^{1}\left(T, \mathbb{R}^{2}\right)$ the canonical semispray $\mathcal{S}=\left(H_{(1) 1}^{(i)}=0, G_{(1) 1}^{(i)}\right)$, where

$$
\begin{aligned}
& G_{(1) 1}^{(1)}=\frac{p r^{3}|V| e^{\frac{2|V| t}{r}}\left(5 r \dot{r}^{-1}-2|V| t \dot{r}^{-1}+|V| r \dot{r}^{-2}\right)-\frac{1}{2} \frac{\partial U}{\partial r}-\frac{m r}{2} \dot{\varphi}^{2}}{m-2 p r^{5}|V| e^{\frac{2|V| t}{r}} \cdot \dot{r}^{-3}} \approx \\
& \approx-\frac{1}{2} \frac{|V|}{r} \dot{r}+\left(\frac{|V| t}{r^{2}}-\frac{5}{2 r}\right) \dot{r}^{2}-\frac{\dot{r}^{3}}{|V|}\left[\frac{5}{3} r^{-1}-\frac{26(|V| t)}{15} r^{-2}+\frac{61(|V| t)^{2}}{120} r^{-3}+\right. \\
& \left.+\frac{(|V| t)^{3}}{180} r^{-4}+\frac{(|V| t)^{4}}{180} r^{-5}+\frac{(|V| t)^{5}}{90} r^{-6}-\frac{(|V| t)^{6}}{45} r^{-7} e^{-\frac{2|V| t}{r}} f\left(\frac{2|V| t}{r}\right)\right]+ \\
& +\frac{m}{4 p|V|} r^{-4} e^{-\frac{2|V| t}{r}} \dot{r}^{3} \dot{\varphi}^{2}, \quad G_{(1) 1}^{(2)}=\frac{\dot{r}}{r} \dot{\varphi} .
\end{aligned}
$$

Proof. The Euler-Lagrange equations of the energy action functional $\mathbf{E}$ are equivalent with the equations

$$
\frac{d^{2} x^{i}}{d t^{2}}+2 H_{(1) 1}^{(i)}\left(t, x^{k}, y_{1}^{k}\right)+2 G_{(1) 1}^{(i)}\left(t, x^{k}, y_{1}^{k}\right)=0, \quad y_{1}^{k}=\frac{d x^{k}}{d t}
$$

where the local geometrical components

$$
H_{(1) 1}^{(i)} \stackrel{\text { def }}{=}-\frac{1}{2} \kappa_{11}^{1}(t) y_{1}^{i}=0
$$

and

$$
G_{(1) 1}^{(i)} \stackrel{\text { def }}{=} \frac{g^{i s}}{4}\left[\frac{\partial^{2} L}{\partial x^{q} \partial y_{1}^{s}} y_{1}^{q}-\frac{\partial L}{\partial x^{s}}+\frac{\partial^{2} L}{\partial t \partial y_{1}^{s}}\right]
$$

represent a semispray on the 1 -jet space $J^{1}\left(T, \mathbb{R}^{2}\right)$.

The polynomial approximate form of the semispray $\mathcal{S}$ produces the canonical nonlinear connection (for more details, see [3] )

$$
\Gamma=\left(M_{(1) 1}^{(i)}=2 H_{(1) 1}^{(i)}=0, N_{(1) j}^{(i)}=\frac{\partial G_{(1) 1}^{(i)}}{\partial y_{1}^{j}}\right) .
$$

Consequently, we can enunciate the following geometrical result:

Corollary 2.2 The canonical nonlinear connection produced by the 2D-monolayer Lagrangian (1.2) has the following approximate spatial components:

$$
\begin{aligned}
& N_{(1) 1}^{(1)}=-\frac{1}{2} \frac{|V|}{r}+\left(\frac{2|V| t}{r^{2}}-\frac{5}{r}\right) \dot{r}-\mathcal{U}(t, r) \dot{r}^{2}+\frac{3 m e^{-\frac{2|V| t}{r}}}{4 p|V| r^{4}} \dot{r}^{2} \dot{\varphi}^{2}, \\
& N_{(1) 2}^{(1)}=\frac{m e^{-\frac{2|V| t}{r}}}{2 p|V| r^{4}} \dot{r}^{3} \dot{\varphi}, \quad N_{(1) 1}^{(2)}=\frac{\dot{\varphi}}{r}, \quad N_{(1) 2}^{(2)}=\frac{\dot{r}}{r}
\end{aligned}
$$


where

$$
\begin{aligned}
\mathcal{U}(t, r)= & \frac{1}{|V|}\left[5 r^{-1}-\frac{26(|V| t)}{5} r^{-2}+\frac{61(|V| t)^{2}}{40} r^{-3}+\frac{(|V| t)^{3}}{60} r^{-4}+\right. \\
& \left.+\frac{(|V| t)^{4}}{60} r^{-5}+\frac{(|V| t)^{5}}{30} r^{-6}-\frac{(|V| t)^{6}}{15} r^{-7} e^{-\frac{2|V| t}{r}} f\left(\frac{2|V| t}{r}\right)\right] .
\end{aligned}
$$

Proof. Some direct computations and the formulas

$$
N_{(1) 1}^{(1)}=\frac{\partial G_{(1) 1}^{(1)}}{\partial \dot{r}}, \quad N_{(1) 2}^{(1)}=\frac{\partial G_{(1) 1}^{(1)}}{\partial \dot{\varphi}}, \quad N_{(1) 1}^{(2)}=\frac{\partial G_{(1) 1}^{(2)}}{\partial \dot{r}}, \quad N_{(1) 2}^{(2)}=\frac{\partial G_{(1) 1}^{(2)}}{\partial \dot{\varphi}}
$$

imply the required result.

\section{The Cartan canonical $\Gamma$-linear connection and its d-torsions}

We use the nonlinear connection from Corollary 2.2 to construct the dual adapted bases of d-vector fields

$$
\left\{\frac{\partial}{\partial t} ; \frac{\delta}{\delta r} ; \frac{\delta}{\delta \varphi} ; \frac{\partial}{\partial \dot{r}} ; \frac{\partial}{\partial \dot{\varphi}}\right\} \subset X(E)
$$

and d-covector fields

$$
\{d t ; d r ; d \varphi ; \delta \dot{r} ; \delta \dot{\varphi}\} \subset X^{*}(E)
$$

where $E=J^{1}\left(T, \mathbb{R}^{2}\right)$, and we set

$$
\begin{aligned}
& \frac{\delta}{\delta r}=\frac{\partial}{\partial r}-N_{(1) 1}^{(1)} \frac{\partial}{\partial \dot{r}}-\frac{\dot{\varphi}}{r} \frac{\partial}{\partial \dot{\varphi}}, \quad \frac{\delta}{\delta \varphi}=\frac{\partial}{\partial \varphi}-\frac{m e^{-\frac{2|V| t}{r}}}{2 p|V| r^{4}} \dot{r}^{3} \dot{\varphi} \frac{\partial}{\partial \dot{r}}-\frac{\dot{r}}{r} \frac{\partial}{\partial \dot{\varphi}}, \\
& \delta \dot{r}=d \dot{r}+N_{(1) 1}^{(1)} d r+\frac{m e^{-\frac{2|V| t}{r}}}{2 p|V| r^{4}} \dot{r}^{3} \dot{\varphi} d \varphi, \quad \delta \dot{\varphi}=d \dot{\varphi}+\frac{\dot{\varphi}}{r} d r+\frac{\dot{r}}{r} d \varphi .
\end{aligned}
$$

Note that, under a change of coordinates (1.1), the elements of the adapted bases (3.1) and (3.2) transform as classical tensors. Consequently, all subsequent geometrical objects on the 1-jet space $J^{1}\left(T, \mathbb{R}^{2}\right)$ (such as the Cartan canonical linear connection, its torsion etc.) will be described in local adapted components.

Using a general result from [3], by direct computations, we give the following important geometrical result:

Proposition 3.1 The Cartan canonical $\Gamma$-linear connection of the $2 D$-monolayer Lagrangian (1.2) has the following approximate adapted components:

$$
C \Gamma=\left(\kappa_{11}^{1}=0, G_{j 1}^{k}, L_{j k}^{i}, C_{j(k)}^{i(1)}\right)
$$

where

$$
\begin{gathered}
G_{j 1}^{k}=\delta_{1}^{k} \cdot \delta_{j 1} \cdot \frac{2 p r^{4}|V|^{2}}{2 p r^{5}|V|-m \dot{r}^{3} e^{-\frac{2|V| t}{r}}}, \\
C_{j(k)}^{i(1)}=\delta_{1}^{i} \cdot \delta_{j 1} \cdot \delta_{k 1} \cdot \frac{3 p r^{5}|V|}{m \dot{r}^{4} e^{-\frac{2|V| t}{r}}-2 p r^{5}|V| \dot{r}}
\end{gathered}
$$

and

$$
\begin{gathered}
L_{11}^{1}=\frac{p r^{3}|V|(2|V| t-5 r)}{m \dot{r}^{3} e^{-\frac{2|V| t}{r}}-2 p r^{5}|V|}-N_{(1) 1}^{(1)} C_{1(1)}^{1(1)} \\
L_{12}^{1}=L_{21}^{1}=-\frac{m e^{-\frac{2|V| t}{r}}}{2 p|V| r^{4}} \dot{r}^{3} \dot{\varphi} \cdot C_{1(1)}^{1(1)}, \quad L_{22}^{1}=\frac{m r}{2 p r^{5}|V| e^{\frac{2|V| t}{r}} \dot{r}^{-3}-m}, \\
L_{11}^{2}=\frac{3 \dot{\varphi}}{2 r \dot{r}}, \quad L_{12}^{2}=L_{21}^{2}=\frac{1}{r}, \quad L_{22}^{2}=0 .
\end{gathered}
$$


Proof. Via the 2D-monolayer adapted derivative operators (3.1), we use the general formulas which give the adapted components of a Cartan canonical connection (see [3]):

$$
\begin{gathered}
G_{j 1}^{k}=\frac{g^{k s}}{2} \frac{\partial g_{s j}}{\partial t}, \quad C_{j(k)}^{i(1)}=\frac{g^{i s}}{2}\left(\frac{\partial g_{j s}}{\partial y_{1}^{k}}+\frac{\partial g_{k s}}{\partial y_{1}^{j}}-\frac{\partial g_{j k}}{\partial y_{1}^{s}}\right)=\frac{g^{i s}}{2} \frac{\partial g_{j s}}{\partial y_{1}^{k}}, \\
L_{j k}^{i}=\frac{g^{i s}}{2}\left(\frac{\delta g_{j s}}{\delta x^{k}}+\frac{\delta g_{k s}}{\delta x^{j}}-\frac{\delta g_{j k}}{\delta x^{s}}\right)= \\
=\Gamma_{j k}^{i}-N_{(1) k}^{(q)} C_{j(q)}^{i(1)}-N_{(1) j}^{(q)} C_{k(q)}^{i(1)}+\frac{g^{i s}}{2} N_{(1) s}^{(q)} \frac{\partial g_{j k}}{\partial y_{1}^{q}}= \\
=\Gamma_{j k}^{i}-N_{(1) k}^{(1)} C_{j(1)}^{i(1)}-N_{(1) j}^{(1)} C_{k(1)}^{i(1)}+\frac{g^{i s}}{2} N_{(1) s}^{(q)} \frac{\partial g_{j k}}{\partial y_{1}^{q}}
\end{gathered}
$$

where

$$
\Gamma_{j k}^{i}=\frac{g^{i s}}{2}\left(\frac{\partial g_{j s}}{\partial x^{k}}+\frac{\partial g_{k s}}{\partial x^{j}}-\frac{\partial g_{j k}}{\partial x^{s}}\right)
$$

and we remind that we have $\left(t, x^{1}, x^{2}, y_{1}^{1}, y_{1}^{2}\right)=(t, r, \varphi, \dot{r}, \dot{\varphi})$.

Remark 3.2 The Cartan canonical connection $C \Gamma$ has the metrical properties

$$
g_{i j / 1}=g_{/ 1}^{i j}=0, \quad g_{i j \mid k}=g_{\mid k}^{i j}=0,\left.\quad g_{i j}\right|_{(k)} ^{(1)}=\left.g^{i j}\right|_{(k)} ^{(1)}=0,
$$

where $", 1 ", " \mid k$, and $\left."\right|_{(k)} ^{(1)}$ " are the T-horizontal, $\mathbb{R}^{2}$-horizontal and vertical covariant derivatives produced by the Cartan linear connection $C \Gamma$ (for more details, see [3]). Consequently, in our jet singletime Lagrange geometrization, the Cartan canonical connection plays a similar role to that of the Levi-Civita connection from classical Riemannian geometry.

Proposition 3.3 The Cartan canonical connection $C \Gamma$ of the 2D-monolayer Lagrangian (1.2) has the next approximate adapted local torsion d-tensors:

$$
\begin{gathered}
T_{1 j}^{k}=\mathcal{P}_{(1) 1(j)}^{(k)(1)}=-G_{j 1}^{k}, \quad P_{i(j)}^{k(1)}=C_{i(j)}^{k(1)}, \\
H_{(1) 1 j}^{(k)}=\delta_{1}^{k} \cdot\left[\delta_{j 1} \cdot\left(\frac{\partial \mathcal{U}}{\partial t} \dot{r}^{2}-\frac{2|V|}{r^{2}} \dot{r}+\frac{3 m e^{-\frac{2|V| t}{r}}}{2 p r^{5}} \dot{r}^{2} \dot{\varphi}^{2}\right)+\delta_{j 2} \cdot \frac{m e^{-\frac{2|V| t}{r}}}{p r^{5}} \dot{r}^{3} \dot{\varphi}\right] \\
R_{(1) 12}^{(1)}=-R_{(1) 21}^{(1)}=\frac{m e^{-\frac{2|V| t}{r}}}{p|V| r^{4}}\left[\frac{3}{2} N_{(1) 1}^{(1)}-\frac{1}{2} \frac{\partial N_{(1) 1}^{(1)}}{\partial \dot{r}}+\left(\frac{1}{r}-\frac{|V| t}{r^{2}}\right) \dot{r}\right] \dot{r}^{2} \dot{\varphi} \\
R_{(1) 12}^{(2)}=-R_{(1) 21}^{(2)}=\frac{1}{r} N_{(1) 1}^{(1)}, \\
P_{(1) 1(1)(1)}^{(1)}=\frac{\partial N_{(1) 1}^{(1)}+R_{(1) 22}^{(1)}=R_{(1) 11}^{(1)}=R_{(1) 22}^{(2)}=0}{\partial \dot{r}} C_{1(1)}^{1(1)}-\frac{p r^{3}|V|(2|V| t-5 r)}{m \dot{r}^{3} e^{-\frac{2|V| t}{r}}-2 p r^{5}|V|}, \\
P_{(1) 1(2)}^{(1)(1)}=P_{(1) 2(1)}^{(1)(1)}=\frac{m e^{-\frac{2|V| t}{r}}}{2 p|V| r^{4}}\left(3+\dot{r} \cdot C_{1(1)}^{1(1)}\right) \dot{r}^{2} \dot{\varphi} \\
P_{(1) 2(2)}^{(1)(1)}=\frac{m e^{-\frac{2|V| t}{r}}}{2 p|V| r^{4} \dot{r}^{-3}}-\frac{m r^{2}}{2 p r^{5}|V| e^{\frac{2|V| t}{r}} \dot{r}^{-3}-m} \\
P_{(1) 1(1)}^{(2)(1)}=-\frac{3 \dot{\varphi}}{2 r \dot{r}}, \quad P_{(1) 1(2)}^{(2)(1)}=P_{(1) 2(1)}^{(2)(1)}=P_{(1) 2(2)}^{(2)(1)}=0 .
\end{gathered}
$$


Proof. Generally, a Cartan canonical connection on the 1-jet space $J^{1}\left(T, \mathbb{R}^{2}\right)$ is characterized by six effective d-tensors of torsion (for more details, see [3]). For our Cartan canonical connection $C \Gamma$ these reduce to the following expressions:

$$
\begin{aligned}
& T_{1 j}^{k}=-G_{j 1}^{k}, \quad H_{(1) 1 j}^{(k)}=-\frac{\partial N_{(1) j}^{(k)}}{\partial t}, \quad R_{(1) i j}^{(k)}=\frac{\delta N_{(1) i}^{(k)}}{\delta x^{j}}-\frac{\delta N_{(1) j}^{(k)}}{\delta x^{i}}, \\
& \mathcal{P}_{(1) 1(j)}^{(k)(1)}=-G_{j 1}^{k}, \quad P_{i(j)}^{k(1)}=C_{i(j)}^{k(1)}, \quad P_{(1) i(j)}^{(k)(1)}=\frac{\partial N_{(1) i}^{(k)}}{\partial y_{1}^{j}}-L_{i j}^{k},
\end{aligned}
$$

where we have $\left(t, x^{1}, x^{2}, y_{1}^{1}, y_{1}^{2}\right)=(t, r, \varphi, \dot{r}, \dot{\varphi})$.

\section{From the dynamics of 2D-monolayer to a jet geometrical Yang-Mills energy}

In monograph [3], using only a given Lagrangian function $L(t, x, y)$ on the 1-jet space $J^{1}\left(\mathbb{R}, M^{n}\right)$, an electromagnetic-like geometrical model was also created. In the background of the electromagnetic-like geometrical formalism from [3], one works with an electromagnetic d-form (this Section the Latin letters run from 1 to $n) \mathbb{F}=F_{(i) j}^{(1)} \delta y_{1}^{i} \wedge d x^{j}$, where

$$
F_{(i) j}^{(1)}=\frac{h^{11}}{2}\left[g_{j s} N_{(1) i}^{(s)}-g_{i s} N_{(1) j}^{(s)}+\left(g_{i q} L_{j s}^{q}-g_{j q} L_{i s}^{q}\right) y_{1}^{s}\right] .
$$

The above electromagnetic components are characterized by the following geometrical Maxwell-like equations (for more details, see [3]):

$$
\begin{aligned}
& F_{(i) j / 1}^{(1)}=\frac{1}{2} \mathcal{A}_{\{i, j\}}\left\{\bar{D}_{(i) 1 \mid j}^{(1)}-D_{(i) s}^{(1)} G_{j 1}^{s}+d_{(i)(s)}^{(1)(1)}\left(\frac{\delta M_{(1) 1}^{(s)}}{\delta x^{j}}-\frac{\delta N_{(1) j}^{(s)}}{\delta t}\right)-\right. \\
& \left.-\left[C_{j(s)}^{l(1)}\left(\frac{\delta M_{(1) 1}^{(s)}}{\delta x^{i}}-\frac{\delta N_{(1) i}^{(s)}}{\delta t}\right)-G_{i 1 \mid j}^{l}\right] h^{11} g_{l q} y_{1}^{q}\right\} \\
& \sum_{\{i, j, k\}} F_{(i) j \mid k}^{(1)}=-\frac{1}{4} \sum_{\{i, j, k\}} \frac{\partial^{3} L}{\partial y_{1}^{i} \partial y_{1}^{l} \partial y_{1}^{s}}\left[\frac{\delta N_{(1) j}^{(s)}}{\delta x^{k}}-\frac{\delta N_{(1) k}^{(s)}}{\delta x^{j}}\right] y_{1}^{l}, \\
& \left.\sum_{\{i, j, k\}} F_{(i) j}^{(1)}\right|_{(k)} ^{(1)}=0,
\end{aligned}
$$

where $\mathcal{A}_{\{i, j\}}$ means an alternate sum, $\sum_{\{i, j, k\}}$ means a cyclic sum, and we have

$$
\begin{gathered}
\bar{D}_{(i) 1}^{(1)}=\frac{h^{11}}{2}\left(\frac{\partial g_{i s}}{\partial t}-M_{(1) 1}^{(q)} \frac{\partial g_{i s}}{\partial y_{1}^{q}}\right) y_{1}^{s}, \quad D_{(i) j}^{(1)}=h^{11} g_{i q}\left[-N_{(1) j}^{(q)}+L_{j s}^{q} y_{1}^{s}\right], \\
d_{(i)(j)}^{(1)(1)}=h^{11}\left[g_{i j}+g_{i q} C_{s(j)}^{q(1)} y_{1}^{s}\right], \\
\bar{D}_{(i) 1 \mid j}^{(1)}=\frac{\delta \bar{D}_{(i) 1}^{(1)}}{\delta x^{j}}-\bar{D}_{(s) 1}^{(1)} L_{i j}^{s}, \quad G_{i 1 \mid j}^{k}=\frac{\delta G_{i 1}^{k}}{\delta x^{j}}+G_{i 1}^{s} L_{s j}^{k}-G_{s 1}^{k} L_{i j}^{s}, \\
F_{(i) j \mid k}^{(1)}=\frac{\delta F_{(i) j}^{(1)}}{\delta x^{k}}-F_{(s) j}^{(1)} L_{i k}^{s}-F_{(i) s}^{(1)} L_{j k}^{s},\left.\quad F_{(i) j}^{(1)}\right|_{(k)} ^{(1)}=\frac{\partial F_{(i) j}^{(1)}}{\partial y_{1}^{k}}-F_{(s) j}^{(1)} C_{i(k)}^{s(1)}-F_{(i) s}^{(1)} C_{j(k)}^{s(1)} .
\end{gathered}
$$


Example 4.1 The Lagrangian function that governs the movement law of a particle of mass $m \neq 0$ and electric charge e, which is concomitantly displaced into an environment endowed both with a gravitational field and an electromagnetic one, is given by (see [8] and [3])

$$
L\left(t, x^{k}, y_{1}^{k}\right)=m c h^{11}(t) \varphi_{i j}\left(x^{k}\right) y_{1}^{i} y_{1}^{j}+\frac{2 e}{m} A_{(i)}^{(1)}\left(x^{k}\right) y_{1}^{i}+\mathcal{F}\left(t, x^{k}\right),
$$

where the semi-Riemannian metric $\varphi_{i j}(x)$ represents the gravitational potentials, $A_{(i)}^{(1)}(x)$ represent the electromagnetic potential, and $\mathcal{F}(t, x)$ is a smooth potential function. In the case of the usual time $\left(\mathbb{R}, h_{11}(t)=1\right)$, applying to the Lagrangian 4.1) our jet geometrical formalism, the electromagnetic components take the form

$$
F_{(i) j}^{(1)}=-\frac{e}{2 m}\left(\frac{\partial A_{(i)}^{(1)}}{\partial x^{j}}-\frac{\partial A_{(j)}^{(1)}}{\partial x^{i}}\right),
$$

and the Maxwell geometrical equations reduce to the classical ones: $\sum_{\{i, j, k\}} F_{(i) j \mid k}^{(1)}=0$, where $\left(\gamma_{j k}^{i}\right.$ are the Christoffel symbols of the semi-Riemannian metric $\varphi_{i j}$ )

$$
F_{(i) j \mid k}^{(1)}=\frac{\partial F_{(i) j}^{(1)}}{\partial x^{k}}-F_{(m) j}^{(1)} \gamma_{i k}^{m}-F_{(i) m}^{(1)} \gamma_{j k}^{m} .
$$

In our opinion, this fact suggests some kind of naturalness attached to our jet single-time Lagrangian geometrical electromagnetic-like theory.

On our particular 1-jet space $J^{1}\left(T, \mathbb{R}^{2}\right)$, the 2D-monolayer Lagrangian (1.2) produces the electromagneticlike 2 -form

$$
\mathbb{F}=F_{(1) 1}^{(1)} \delta \dot{r} \wedge d r+F_{(1) 2}^{(1)} \delta \dot{r} \wedge d \varphi+F_{(2) 1}^{(1)} \delta \dot{\varphi} \wedge d r+F_{(2) 2}^{(1)} \delta \dot{\varphi} \wedge d \varphi
$$

where $F_{(1) 1}^{(1)}=F_{(2) 2}^{(1)}=0$ and

$$
F_{(2) 1}^{(1)}=-F_{(1) 2}^{(1)}=\frac{1}{2}\left[\frac{3 m r}{2}+\frac{m^{2} e^{-\frac{2|V| t}{r}}}{4 p|V| r^{4}} \cdot \dot{r}^{3}\right] \cdot \dot{\varphi} .
$$

The magnitude (i.e., the jet geometrical Yang-Mills energy) of our theoretical electromagnetic-like field $\mathbb{F}$ produced by the 2D-monolayer Lagrangian (1.2) is defined by the formula

$$
\operatorname{Ey\mathcal {M}}(\mathbb{F})=\frac{1}{2 m} \cdot \operatorname{Trace}\left[\mathbf{F} \cdot{ }^{T} \mathbf{F}\right]=\frac{1}{m} \cdot\left[F_{(1) 2}^{(1)}\right]^{2},
$$

where the skew-symmetric electromagnetic matrix is

$$
\mathbf{F}=\left(\begin{array}{cc}
0 & F_{(1) 2}^{(1)} \\
-F_{(1) 2}^{(1)} & 0
\end{array}\right) \in o(2)=L(O(2)) .
$$

Note that the matrix $\mathbf{F}$ belongs to the Lie algebra $o(2)$ of the Lie group of orthogonal matrices

$$
O(2)=\left\{A \in G L_{2}(\mathbb{R}) \mid A \cdot{ }^{T} A=I_{2}\right\} .
$$

The jet geometrical Yang-Mills energy produced by the 2D-monolayer Lagrangian (1.2) cancels iff

$$
F_{(1) 2}^{(1)}=0 \Leftrightarrow\left[\frac{3 m r}{2}+\frac{m^{2} e^{-\frac{2|V| t}{r}}}{4 p|V| r^{4}} \cdot \dot{r}^{3}\right] \cdot \dot{\varphi}=0 .
$$




\section{Jacobi equations for deviation of geodesics from an instanton-like so- lution. Computer imaging simulations and physical interpretations}

Let us find the singular trajectories of the differential equations from the Proof of Proposition 2.1;

$$
\frac{d y_{1}^{i}}{d t}+2 G_{(1) 1}^{(i)}\left(t, x^{k}, y_{1}^{k}\right)=0, \quad \frac{d x^{k}}{d t}=y_{1}^{k}
$$

with

$$
G_{(1) 1}^{(i)}=\frac{g^{i s}}{4}\left[\frac{\partial^{2} L}{\partial x^{q} \partial y_{1}^{s}} y_{1}^{q}-\frac{\partial L}{\partial x^{s}}+\frac{\partial^{2} L}{\partial t \partial y_{1}^{s}}\right]
$$

where

$$
G_{(1) 1}^{(1)}=\frac{p r^{3}|V| e^{\frac{2|V| t}{r}}\left(5 r \dot{r}^{-1}-2|V| t \dot{r}^{-1}+|V| r \dot{r}^{-2}\right)-\frac{1}{2} \frac{\partial U}{\partial r}-\frac{m r}{2} \dot{\varphi}^{2}}{m-2 p r^{5}|V| e^{\frac{2|V| t}{r}} \cdot \dot{r}^{-3}}, \quad G_{(1) 1}^{(2)}=\frac{\dot{r}}{r} \dot{\varphi} .
$$

One can note that a singularity in (5.3) represents itself a zero difference of the kinetic energy and a part of the potential energy:

$$
\frac{m}{2} \dot{r}^{2}+\frac{m r^{2}}{2} \dot{\varphi}^{2}-p r^{5}|V| e^{\frac{2|V| t}{r}} \cdot \dot{r}^{-1}, \quad \dot{\varphi} \rightarrow 0 .
$$

Therefore, in the neighbourhood of singular behavior of $G_{(1) 1}^{(1)}$, the effects stipulated by a rest $U$ of the potential energy $U_{s}$ are small. Consequently, these above allow us to make the proposal that the Lagrangian $L$ can be expanded into series $L=L_{0}+\delta L$, where the unperturbed Lagrangian $L_{0}$ gives trajectories being nearby the trajectories with zero Hamiltonian:

$$
E_{\text {inst }}=\frac{m}{2} \dot{r}^{2}+\frac{m r^{2}}{2} \dot{\varphi}^{2}+p r^{5}|V| e^{\frac{2|V| t}{r}} \cdot \dot{r}^{-1}-U=0 .
$$

A solution which obeys condition (5.5) is called an instanton-like solution. In our case, the geodesic trajectory of a particle in 2D-membrane is closed to the instanton-like solution $\overrightarrow{r_{0}}$. It has been shown in [5], 4] that a calculation of the trajectories having zero energy in a field of the electrocapillarity forces of monolayer can be performed in a resonant approximation in form of a correction $\overrightarrow{\delta r}$ for $\overrightarrow{r_{0}}$. The correction $\overrightarrow{\delta r}$ is described by some Jacobi equations which are equivalent to some equations that describe the physical system under the action of the field having the frequency closed to an eigenfrequency of the system. Such type of action is called a resonant action. Now, let us simulate 2 the trajectories described by the equations (5.1-5.3). These trajectories are represented in Fig. 1. According to images in the figure steady states are limiting cycles, and a relaxation to the limiting cycle is radial one with $\dot{\varphi} \rightarrow 0$ for the large speeds. Hence, the electrocapillar action on the system under consideration holds a spherical symmetry.

Therefore, as resonant action on a particle of the monolayer one chooses a geometrical Yang-Mills field with zero energy and the symmetry group $O(2)$, that was proposed in Section 4 ;

$$
F_{(1) 2}^{(1)} \stackrel{\text { def }}{=}\left[\frac{3 m r}{2}+\frac{m^{2} e^{\frac{-2|V| t}{r}}}{4 p|V| r^{4}} \cdot \dot{r}^{3}\right]=0 .
$$

Now, we can find the trajectory of a particle in the field (5.6). To do that, it is necessary to make a change $t \rightarrow-t, \dot{r} \rightarrow-\dot{r}$ because the centrifugal acceleration of the particle of monolayer has opposite direction with respect to the centripetal acceleration produced by this field. After this change one gets that the components $F_{(1) 2}^{(1)}(-t)$ are proportional to

$$
\left[\frac{3 m r_{0}}{2}-\frac{m^{2} e^{\frac{2|V| t}{r_{0}}}}{4 p|V| r_{0}^{4}} \cdot{\dot{r_{0}}}^{3}\right]=0
$$

\footnotetext{
${ }^{2}$ Please note that the four computer graphic simulations used and cited in this preliminary version of our paper are missing because of some uploading problems. If some researcher is interested in these graphics, we warmly and cordially invite him to contact any author of this research work.
} 
and the corresponding trajectories are:

$$
\frac{3 r_{0}}{2}-\frac{m e^{\frac{2|V| t}{r_{0}}}}{4 p|V| r_{0}^{4}} \cdot{\dot{r_{0}}}^{3}=0 \quad \text { for arbitrary } \quad \dot{\varphi_{0}} .
$$

Comparing the expressions (5.4) and (5.8), one can conclude that the trajectory of a monolayer particle in the geometrical Yang-Mills field (5.6) with zero energy is closed to the solution of Eqs. (5.1, 5.2, 5.3). Hence, the physical meaning of the introduced geometrical field (5.6) is that this field is a field of the electrocapillarity forces which acts resonantly on monolayer molecules.

Generally, the instanton-like solution is an unstable state of perturbation, which is a transition from one to at least two stable states. In our case, the perturbation describes a first-order phase transition, and we will mean this transition phase as an exit of the system from the resonance with the Yang-Mills field. Further we will describe a resonance detuning. This process is a deviation $3 \overrightarrow{\delta r}=\{\delta r(t), \delta \varphi(t)\}$ of a solution of Eq. (5.1) along the trajectory $\vec{r}_{0}=\left\{\delta r_{0}, \delta \varphi_{0}\right\}$ (5.8) of the zero Yang-Mills field (5.6):

$$
\vec{r}(t)=\vec{r}_{0}(-t)+\overrightarrow{\delta r}(t) .
$$

Now, let us find a Hamiltonian of the system under above considerations, taking into account that

$$
L=\frac{1}{2} m \dot{r}^{2}+U(r, t)+\frac{1}{2} m r^{2} \dot{\varphi}^{2}-\frac{p|V| r^{5} e^{\frac{2 t|V|}{r}}}{\dot{r}},
$$

where $U(r, t)$ is given by (1.3), and the non-zero components of the metric tensor are

$$
g_{11}=\frac{1}{2}\left(m-\frac{2 p|V| r^{5} e^{\frac{2 t V}{r}}}{\dot{r}^{3}}\right), \quad g_{22}=\frac{1}{2} m r^{2} .
$$

Let us use instead of classical Hamiltonian the difference between $g_{11} \dot{r}^{2}+g_{22} \dot{\varphi}^{2}$ and the given Lagrangian. It follows that our renormalized Hamiltonian reads

$$
\begin{aligned}
& H=g_{11} \dot{r}^{2}+g_{22} \dot{\varphi}^{2}-L= \\
& =-p\left[e^{\frac{2 t|V|}{r}}\left(\frac{1}{45} t^{4}|V|^{4} r+\frac{1}{45} t^{3}|V|^{3} r^{2}+\frac{1}{30} t^{2}|V|^{2} r^{3}+\frac{16}{15} t|V| r^{4}-\frac{4 r^{5}}{3}+\frac{2 t^{5}|V|^{5}}{45}\right)-\right. \\
& \left.-\frac{4 t^{6}|V|^{6}}{45 r} f\left(\frac{2 t|V|}{r}\right)\right]+\frac{1}{2} \dot{r}^{2}\left(m-\frac{2 p|V| r^{5} e^{\frac{2 t|V|}{r}}}{\dot{r}^{3}}\right)-\frac{1}{2} m \dot{r}^{2}+\frac{p|V| r^{5} e^{\frac{2 t|V|}{r}}}{\dot{r}} .
\end{aligned}
$$

Let us consider now that the unperturbed renormalized Hamiltonian is the Yang-Mills energy

$$
H_{Y M}=\frac{\dot{\varphi}^{2}\left(\frac{m^{2} e^{-\frac{2 t|V|}{r}}}{4 p|V| r^{4}}+\frac{3}{2} m r\right)^{2}}{4 m} .
$$

Then, the perturbation is determined as

$$
\delta L=-\Delta H=-\left(H-H_{Y M}\right)=-\left[-U(r, t)-\frac{m \dot{\varphi}^{2} e^{-\frac{4 t|V|}{r}}\left(m \dot{r}^{3}+6 p|V| r^{5} e^{\frac{2 t|V|}{r(t)}}\right)^{2}}{64 p^{2}|V|^{2} r^{8}}\right] .
$$

It follows that the unperturbed Lagrangian is

$$
L_{0}=g_{22} \dot{\phi}^{2}+g_{11} \dot{r}^{2}-H_{Y M}=-\frac{\dot{\varphi}^{2}\left(\frac{m^{2} e^{-\frac{2 t|V|}{r}} \dot{r}^{3}}{4 p|V| r^{4}}+\frac{3}{2} m r\right)^{2}}{4 m}+\frac{1}{2} m r^{2} \dot{\varphi}^{2}+\frac{1}{2} \dot{r}^{2}\left(m-\frac{2 p|V| r^{5} e^{\frac{2 t|V|}{r}}}{\dot{r}^{3}}\right) .
$$

\footnotetext{
${ }^{3}$ Please note that here $\delta$ means a small variation. This does not represent the covariant derivative $\delta$ used in the previous geometrical construction of distinguished 1-forms $\delta \dot{r}$ and $\delta \dot{\varphi}$.
} 
Let us determine now an unperturbed semispray $G_{0(1) 1}^{i}$ as a semispray satisfying the following system of equations:

$$
\frac{d y_{0}^{i}}{d t}+2 G_{0}^{(i)}\left(t, x_{0}^{k}, y_{01}^{k}\right)=0, \quad \frac{d x_{0}^{k}}{d t}=y_{01}^{k},
$$

where

$$
\begin{aligned}
G_{0}^{(i)}(i) 1 & =\left.\frac{g^{i s}}{4}\left[\frac{\partial^{2}\left(L_{0}+\delta L\right)}{\partial x^{q} \partial y_{1}^{s}} y_{1}^{q}-\frac{\partial\left(L_{0}+\delta L\right)}{\partial x^{s}}+\frac{\partial^{2}\left(L_{0}+\delta L\right)}{\partial t \partial y_{1}^{s}}\right]\right|_{\vec{r}_{0}, \vec{r}_{0}} \equiv \\
& \equiv \frac{g^{i s}}{4}\left[\frac{\partial^{2} L_{0}}{\partial x^{q} \partial y_{1}^{s}} y_{1}^{q}-\frac{\partial L_{0}}{\partial x^{s}}+\frac{\partial^{2} L_{0}}{\partial t \partial y_{1}^{s}}\right]+\mathcal{O}\left(d^{0} \delta L\right) .
\end{aligned}
$$

A calculation of a perturbation $\delta G_{(1) 1}^{i}$ of the semispray $G_{(1) 1}^{i}=G_{0(1) 1}^{i}+\delta G_{(1) 1}^{i}$ gives the expression

$$
\delta G_{(1) 1}^{i}=\frac{g^{i s}}{4}\left[\frac{\partial^{2} L_{0}}{\partial x^{q} \partial y_{1}^{s}} \delta y_{1}^{q}+\frac{\partial^{2} \delta L}{\partial x^{q} \partial y_{1}^{s}} y_{1}^{q}-\frac{\partial \delta L}{\partial x^{s}}+\frac{\partial^{2} \delta L}{\partial t \partial y_{1}^{s}}\right]-\mathcal{O}\left(d^{0} \delta L\right),
$$

which leads to equations

$$
\begin{gathered}
\frac{d \delta y_{1}^{i}}{d t}+\left.\frac{g^{i s}}{2} \frac{\partial^{2} L_{0}}{\partial x^{q} \partial y_{1}^{s}}\right|_{\vec{r}_{0}, \vec{r}_{0}} \delta y_{1}^{q}+ \\
+\frac{g^{i s}}{2}\left\{\left.\left[\frac{\partial^{2}}{\partial x^{q} \partial y_{1}^{s}}\left(\frac{\partial \delta L}{\partial x^{\kappa}}\right)\right] y_{1}^{q}\right|_{\vec{r}_{0}, \vec{r}_{0}} \delta x^{\kappa}+\left.\left[\frac{\partial^{2}}{\partial x^{q} \partial y_{1}^{s}}\left(\frac{\partial \delta L}{\partial y_{1}^{\kappa}}\right)\right] y_{1}^{q}\right|_{\vec{r}_{0}, \vec{r}_{0}} \delta y_{1}^{\kappa}-\left.\left[\frac{\partial}{\partial x^{s}} \frac{\partial \delta L}{\partial x^{\kappa}}\right]\right|_{\vec{r}_{0}, \vec{r}_{0}} \delta x^{\kappa}-\right. \\
\left.-\left.\left[\frac{\partial}{\partial x^{s}} \frac{\partial \delta L}{\partial y_{1}^{\kappa}}\right]\right|_{\vec{r}_{0}, \vec{r}_{0}} \delta y_{1}^{\kappa}+\left.\left[\frac{\partial^{2}}{\partial t \partial y_{1}^{s}}\left(\frac{\partial \delta L}{\partial x^{\kappa}}\right)\right]\right|_{\vec{r}_{0}, \vec{r}_{0}} \delta x^{\kappa}+\left.\left[\frac{\partial^{2}}{\partial t \partial y_{1}^{s}}\left(\frac{\partial \delta L}{\partial y_{1}^{\kappa}}\right)\right]\right|_{\vec{r}_{0}, \vec{r}_{0}} \delta y_{1}^{\kappa}\right\}=0, \\
\frac{d \delta x^{k}}{d t}=\delta y_{1}^{k} .
\end{gathered}
$$

Since due to condition (5.4) $\dot{\varphi}_{0}=\epsilon$ is very small: $\dot{\varphi}_{0}=\epsilon \ll 1$ and $\delta L$ depends quadratically on $\dot{\varphi}$, then the variables in (5.14, 5.15) are separated:

$$
\begin{gathered}
\frac{d \delta y_{1}^{2}}{d t}+\left.\frac{g^{22}}{2}\left\{\left[\frac{\partial^{2}}{\partial x^{1} \partial y_{1}^{2}}\left(\frac{\partial \delta L}{\partial y_{1}^{2}}\right)\right] y_{1}^{1}+\left[\frac{\partial^{2}}{\partial t \partial y_{1}^{2}}\left(\frac{\partial \delta L}{\partial y_{1}^{2}}\right)\right]\right\}\right|_{r_{0}, \dot{r}_{0}} \delta y_{1}^{2}=0, \\
\frac{d \delta y_{1}^{1}}{d t}-\left.\frac{g^{11}}{2}\left[\frac{\partial}{\partial x^{1}} \frac{\partial \delta L}{\partial x^{1}}\right]\right|_{r_{0}, \dot{r}_{0}} \delta x^{1}+\left.\frac{g^{11}}{2}\left\{\left[\frac{\partial^{2}}{\partial x^{q} \partial y_{1}^{1}}\left(\frac{\partial \delta L}{\partial y_{1}^{2}}\right)\right] y_{1}^{q}+\left[\frac{\partial^{2}}{\partial t \partial y_{1}^{1}}\left(\frac{\partial \delta L}{\partial y_{1}^{2}}\right)\right]\right\}\right|_{r_{0}, \delta x^{2}, \dot{r}_{0}, \delta y_{1}^{2}} \delta y_{1}^{2}+ \\
+\left.\frac{g^{11}}{2} \frac{\partial^{2} L_{0}}{\partial x^{1} \partial y_{1}^{1}}\right|_{\vec{r}_{0}, \vec{r}_{0}} \delta y_{1}^{1}=0, \quad \frac{d \delta x^{k}}{d t}=\delta y_{1}^{k} .
\end{gathered}
$$

Let us examine the case $\dot{\varphi}_{0}=\epsilon=0$. In this case, a full separation of variables takes place:

$$
\begin{aligned}
& \frac{d \delta y_{1}^{2}}{d t}+\left.\frac{g^{22}}{2}\left\{\left[\frac{\partial^{2}}{\partial x^{1} \partial y_{1}^{2}}\left(\frac{\partial \delta L}{\partial y_{1}^{2}}\right)\right] y_{1}^{1}+\left[\frac{\partial^{2}}{\partial t \partial y_{1}^{2}}\left(\frac{\partial \delta L}{\partial y_{1}^{2}}\right)\right]\right\}\right|_{r_{0}, \dot{r}_{0}} \delta y_{1}^{2}=0 \\
& \frac{d \delta y_{1}^{1}}{d t}-\left.\frac{g^{11}}{2}\left[\frac{\partial}{\partial x^{1}} \frac{\partial \delta L}{\partial x^{1}}\right]\right|_{r_{0}, \dot{r}_{0}} \delta x^{1}+\left.\frac{g^{11}}{2} \frac{\partial^{2} L_{0}}{\partial x^{1} \partial y_{1}^{1}}\right|_{\vec{r}_{0}, \vec{r}_{0}} \delta y_{1}^{1}=0, \\
& \frac{d \delta x^{k}}{d t}=\delta y_{1}^{k} .
\end{aligned}
$$

Substituting (5.10) and (5.11) into (5.16 - 5.18) and making some simple transformations, one gets the equations for deviation in explicit form:

$$
\begin{aligned}
\left(2 p|V| r_{0}^{5} e^{\frac{2 t|V|}{r_{0}}}-m \dot{r}_{0}^{3}\right) \frac{d^{2}}{d t^{2}} \delta r(t)+p|V| r_{0}^{3} e^{\frac{2 t|V|}{r_{0}}}\left(2 t|V|-5 r_{0}\right) \dot{r}_{0} \frac{d}{d t} \delta r(t)+\dot{r}_{0}^{3} \ddot{U}\left(t, r_{0}\right) \delta r & =0, \\
\frac{d^{2}}{d t^{2}} \delta \varphi(t)+\frac{\dot{r}_{0}\left(m \dot{r}_{0}^{3}+6 p|V| r_{0}^{5} e^{\frac{2 t|V|}{r_{0}}}\right)\left[m\left(t|V|-2 r_{0}\right) \dot{r}_{0}^{3}+3 p|V| r_{0}^{6} e^{\frac{2 t|V|}{r_{0}}}\right]}{8 p^{2}|V|^{2} r_{0}^{12} \exp \left\{4 t|V| / r_{0}\right\}} \frac{d}{d t} \delta \varphi(t) & =0 .
\end{aligned}
$$


According to equation (5.8) the resonant solution $r_{0}(-t)$ satisfies the equation

$$
m \dot{r}_{0}^{3}+6 p|V| r_{0}^{5} e^{\frac{2 t|V|}{r_{0}}}=0
$$

The substitution of (5.21) into (5.20) gives the equation

$$
\frac{d^{2}}{d t^{2}} \delta \varphi(t)=0
$$

that can be easy solved as

$$
\delta \varphi=C_{1}+C_{2} t
$$

Further a simulation will be performed. Let us choose the following particular values for our parameters: $p=10,|V|=1000, m=1$ and assume that the constants in (5.22) are equal to $C_{1}=0, C_{2}=1$. Using the physical condition $r_{0} \rightarrow R_{0}-|V| t \rightarrow 0$ for large time $t$, the resonant solution $r_{0}(-t)$ has been found approximately by a transformation of the equation (5.8) for large time $t$ to the form

$$
m \dot{r}_{0}^{3}+6 p|V| r_{0}^{5} e^{2|V| t /\left(R_{0}-|V| t\right)}=0, \quad \text { for large } t .
$$

The solution of equation (5.23) has the form

$$
\begin{aligned}
& r_{0}=27 \sqrt{m} R_{0} v e^{\frac{t|V|}{t|V|-R_{0}}}\left\{-4 \sqrt[3]{6} \sqrt[3]{p} R_{0}^{5 / 3} e^{-\frac{2 R_{0} / 3}{R_{0}-t|V|}}\left[f\left(\frac{2}{3}\right)-f\left(\frac{2 R_{0} / 3}{R_{0}-t|V|}\right)\right]\right. \\
& \left.+e^{-\frac{2 t|V| / 3}{R_{0}-t|V|}}\left(9 \sqrt[3]{m}|V|^{2 / 3}+6 \sqrt[3]{6} \sqrt[3]{p} R_{0}^{5 / 3}\right)-6 \sqrt[3]{6} \sqrt[3]{p} R_{0}{ }^{2 / 3}\left(R_{0}-t|V|\right)\right\}^{-3 / 2} \quad \text { for large } t .
\end{aligned}
$$

The simulation of the case with $\epsilon=0$ results from Figs. 2-4.

Finally, we consider it is important to note that the obtained results are correlated with a behavior of the surface tension $P$ of the monolayer. Moreover, the dependence $r(t)$ shown in Fig. 4 behaves as an isotherm $P(S)$. It has a plateau corresponding to the first-order phase transition and for large $t$ to a fold that corresponds to the collapse of monolayer.

Acknowledgements. The present work was developed under the auspices of Grant 1196/2012 BRFFR-RA F12RA-002, within the cooperation framework between Romanian Academy and Belarusian Republican Foundation for Fundamental Research.

A version of this paper was presented at VIII-th International Conference "Finsler Extensions of Relativity Theory“, June 25 - July 1, 2012, Moscow-Fryazino, Russia.

\section{References}

[1] S. Acharya, J.P. Hill, K. Ariga, Soft Langmuir-Blodgett Technique for Hard Nanomaterials, Adv. Mater., vol. 21 (2009), 2959-2981.

[2] G.S. Asanov, Jet extension of Finslerian gauge approach, Fortschritte der Physik, vol. 38, no. 8 (1990), 571-610.

[3] V. Balan, M. Neagu, Jet Single-Time Lagrange Geometry and Its Applications, John Wiley \& Sons, Inc., Hoboken, New Jersey, 2011.

[4] H.V. Grushevskaya, N.G. Krylova, Application of Finsler geometry to instanton-like process of LBnanostructures emerging in monolayer, In: Proc. of VIII-th International Bolyai - Gauss - Lobachevsky Conference on Non-Euclidean Geometry in Modern Physics and Mathematics, 22 - 25 May 2012, Uzhgorod, Ukraine. Ed. A.M. Zavilopulo. (Institute of electronic physics, Uzhgorod, 2012). P.44.

[5] H.V. Grushevskaya, N.G. Krylova, Effects of Finsler geometry in physics surface phenomena: case of monolayer systems, Hypercomplex Numbers in Geometry and Physics 1 (15), vol. 8 (2011), 128-146.

[6] V.V. Hrushevsky, H.V. Krylova, Thermodynamics of phase states in Langmuir Blodgett monolayers, In: "Low-dimensional systems-2" (Eds. S.A. Maskevich et al.), Grodno State University, Grodno, no. 4 (2005), 30-36. 
[7] V.M. Kaganer, E.B. Loginov, Phys. Rev., E 51 (1995), 2237.

[8] R. Miron, M. Anastasiei, The Geometry of Lagrange Spaces: Theory and Applications, Kluwer Academic Publishers, Dordrecht, 1994.

[9] H. Motschmann, H. Möhwald, Langmuir-Blodgett Films, Handbook of Applied Surface and Colloid Chemistry (Ed. Krister Holmberg), Ch. 28 (2001), 629-648.

[10] M. Neagu, Riemann-Lagrange Geometry on 1-Jet Spaces, Matrix Rom, Bucharest, 2005.

[11] P.J. Olver, Applications of Lie Groups to Differential Equations, Springer-Verlag, New-York, 1986. 